\title{
METHODS REPORT
}

\section{A new assay for tRNA aminoacylation kinetics}

\author{
ALEXEY D. WOLFSON, JEFFREY A. PLEISS, and OLKE C. UHLENBECK \\ Department of Chemistry and Biochemistry, University of Colorado, Boulder, Colorado 80309-0215, USA
}

\begin{abstract}
An improved quantitative assay for tRNA aminoacylation is presented based on charging of a nicked tRNA followed by separation of an aminoacylated $3^{\prime}$-fragment on an acidic denaturing polyacrylamide gel. Kinetic parameters of tRNA aminoacylation by Escherichia coli AlaRS obtained by the new method are in excellent agreement with those measured by the conventional method. This assay provides several advantages over the traditional methods of measuring tRNA aminoacylation: (1) the fraction of aminoacyl-tRNA is measured directly; (2) data can be obtained at saturating amino acid concentrations; and (3) the assay is significantly more sensitive.
\end{abstract}

Keywords: acid gel electrophoresis; alanine; aminoacyl-tRNA synthetase; tRNA, kinetics

\section{INTRODUCTION}

The availability of co-crystal structures of several tRNAs bound to their cognate aminoacyl tRNA synthetases (Cusack, 1995, 1997; Arnez \& Moras, 1997) permits the design of biochemical experiments aimed at understanding how specific binding of the tRNA by the enzyme is achieved and how interactions with the tRNA affect the catalytic reaction mechanism (Yarus, 1988). Because such "structure-function" experiments require determining the aminoacylation kinetics of a variety of modified tRNAs and mutant enzymes, it is critical to have a reliable assay. Although excellent quantitative assays are available that measure the first, amino acid adenylation, step of the reaction (Fersht et al., 1975; Mazat et al., 1982), the primary assay available for measuring the transfer of the amino acid onto the tRNA has several shortcomings. In its most common format, this assay determines the amount of tritiated amino acid covalently linked to the tRNA, and then calculates the fraction of tRNA aminoacylated using the input tRNA concentration (Loftfield, 1972; Eigner \& Loftfield, 1974). Because both the amino acid and the tRNA are expected to help organize the active site of a synthetase, their mutual presence on the enzyme is desirable for many kinetic measurements. However, it is often impractical to perform reactions under saturating amino acid concentrations. Several aminoacyl-tRNA synthetases have $K_{M}$ values for amino acid in the range of

Reprint requests to: Olke C. Uhlenbeck, Department of Chemistry and Biochemistry, University of Colorado, Boulder, Colorado 803090215, USA; e-mail: olke.uhlenbeck@colorado.edu.
200-400 $\mu \mathrm{M}$ (Hill \& Schimmel, 1989; Eriani et al., 1993; Ibba et al., 1996), whereas the corresponding $K_{M}$ values for tRNA are between 0.2 and $2 \mu \mathrm{M}$. Because of this, at saturating amino acid concentration, prohibitive amounts of radioactivity would be required to measure aminoacylation of subsaturating concentrations of tRNA. The weak binding of the amino acid and the tight binding of the tRNA by synthetases also prevent performing pre-steady-state kinetics on this phase of the reaction-a necessary requirement for dissecting the tRNA binding step in the reaction mechanism. Finally, by not measuring aminoacylation of tRNA directly, the conventional assay cannot detect the presence of inactive tRNA molecules in the reaction, which is a possibility when mutant tRNAs are used.

Here we demonstrate the feasibility of an assay that directly measures the fraction of aminoacylated tRNA by its altered mobility on an acidic, denaturing polyacrylamide gel. Such gels have been used to estimate the levels of aminoacylated tRNA in vivo (Varshney et al., 1991; McClain et al., 1998), and to detect selfaminoacylation by a ribozyme (Illangasekare et al., 1997). However, initial experiments revealed that the resolution of these gels was not sufficient to obtain accurate kinetic data when intact tRNA was used. Thus, we employed a tRNA made of two RNA fragments with a nick at a position near the $3^{\prime}$ terminus that does not affect aminoacylation kinetics (Liu \& Musier-Forsyth, 1994). Because this assay uses high-specific activity $\left[{ }^{32} \mathrm{P}\right]-$ labeled tRNA and measures aminoacylation directly, it overcomes the major shortcomings of the tritiated amino acid assay and should be valuable for structure-function studies. 
A

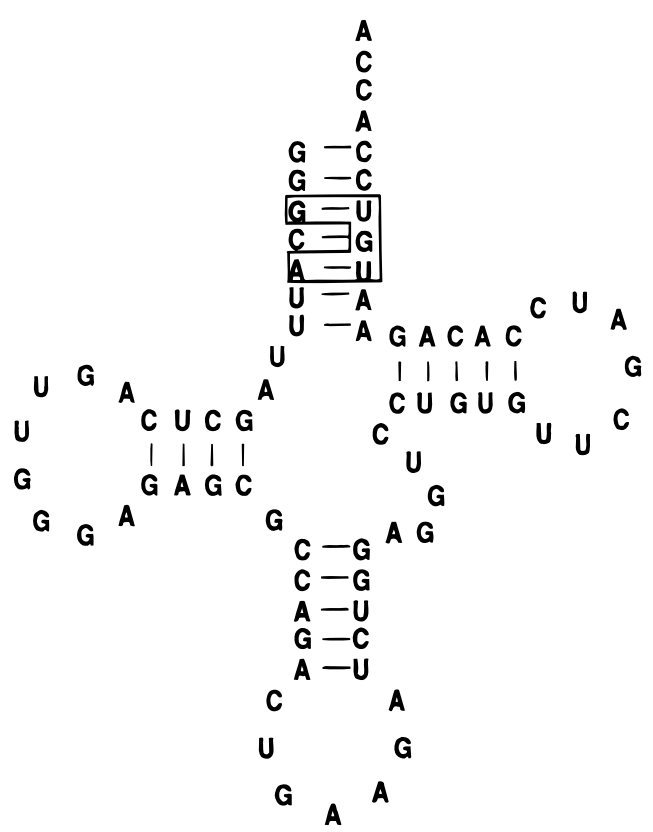

B

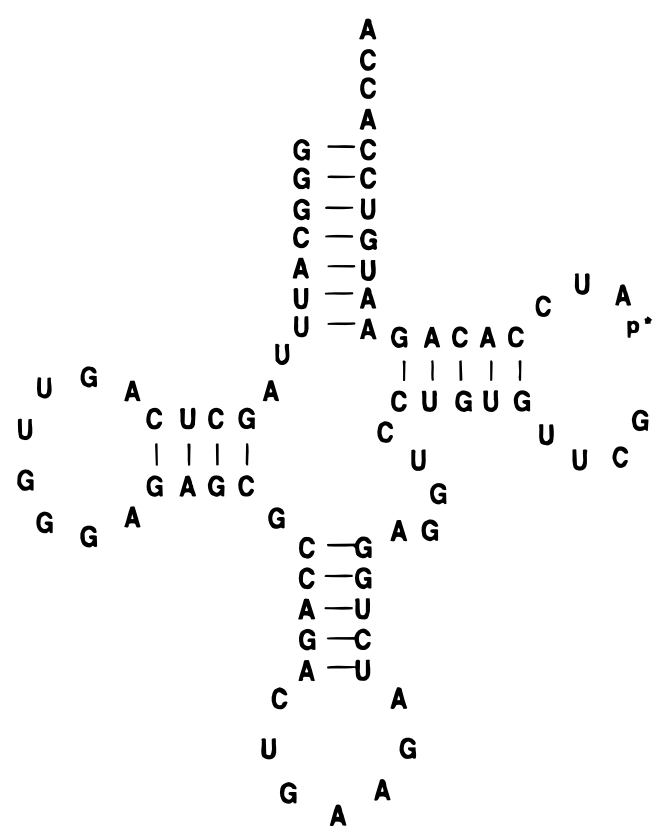

FIGURE 1. A: Structure of the YFA2 tRNA. Nucleotides changed from the wild-type yeast tRNA ${ }^{\text {Phe }}$ are boxed. B: Structure of the nicked YFA2. Position of the $5^{\prime}$ phosphate used for the labeling is indicated.

\section{RESULTS AND DISCUSSION}

The tRNA used in this work is a derivative of yeast tRNA $^{\text {Phe }}$ that contains several acceptor stem mutations providing it with the recognition nucleotides of Escherichia coli alanine tRNA synthetase (Fig. 1A). As will be shown elsewhere, this tRNA is fully active for aminoacylation by both yeast PheRS and E. coli AlaRS. A bimolecular version of this dual-specificity tRNA (Fig. 1B) was prepared by combining a 57-nt $5^{\prime}$ fragment made by transcription with a 19-nt 3' fragment made by chemical synthesis. When equimolar amounts of the two oligonucleotides were annealed, nondenaturing gels revealed that more than $90 \%$ of a radiolabeled $3^{\prime}$ fragment comigrated with intact tRNA (data not shown). The resulting bimolecular tRNA contains a nick in the phosphate backbone between residues 57 and 58, a site previously shown not to alter tRNA folding greatly (Pan et al., 1991). Conventional aminoacylation experiments reveal that the nicked tRNA has the same $k_{\text {cat }} / K_{M}$ for aminoacylation as the intact tRNA with yeast PheRS, and only a fivefold lower $k_{c a t} / K_{M}$ with $E$. coli AlaRS (data not shown). These results are not affected by the presence of a phosphate on the $5^{\prime}$ terminus of the 19-mer. Comparable results have been obtained using $E$. coli tRNA ${ }^{\text {Pro }}$ containing a nick at the same site (Liu \& Musier-Forsyth, 1994).

The acid gel assay is performed using the nicked tRNA substrate where the 19 -mer is $5^{\prime}-\left[{ }^{32} \mathrm{P}\right]$ labeled. Figure $2 \mathrm{~A}$ shows a gel analyzing the products of a time course of aminoacylation of this substrate by AlaRS.
Separation of the aminoacyl oligonucleotide product from the unaminoacylated substrate is excellent and easily quantified. Equally good separation is observed when the substrate is aminoacylated by PheRS, suggesting that the identity of the amino acid does not greatly effect separation (data not shown). Because a small amount of "smearing" was seen on the gel, there was a concern that spontaneous deacylation of the oligonucleotide occurred during the long (up to $14 \mathrm{~h}$ ) electrophoresis time despite the low $\mathrm{pH}$ of the gel buffer. However, control experiments using $\left[{ }^{3} \mathrm{H}\right]$-labeled aminoacyl tRNA revealed less than $5 \%$ deacylation upon incubation in the gel buffer for $24 \mathrm{~h}$ at $4{ }^{\circ} \mathrm{C}$. Thus, the gel accurately reflects the fraction of aminoacylated 19mer when the time point was taken.

In order to compare the acid gel assay with the conventional assay, a double-label experiment was performed using $5^{\prime}-\left[{ }^{32} \mathrm{P}\right]$-labeled 19 -mer and $\left[{ }^{3} \mathrm{H}\right]$-alanine. Duplicate samples were taken at various times and analyzed both on acid gels and by the tRNA precipitation assay. A sample time course is shown in Figure 2B. Although the reaction rates determined by the two assays are in good agreement, the extent of the reaction is significantly lower in the tritium assay. This disparity is likely due to the inaccuracy associated with calculating the aminoacylated tRNA concentration from the acid-insoluble radioactivity. Although the experiment made use of a careful independent determination of the tritium-counting efficiency in the format of the assay, several uncontrollable variables, including the presence of free amino acid in the enzyme, contaminating 
A

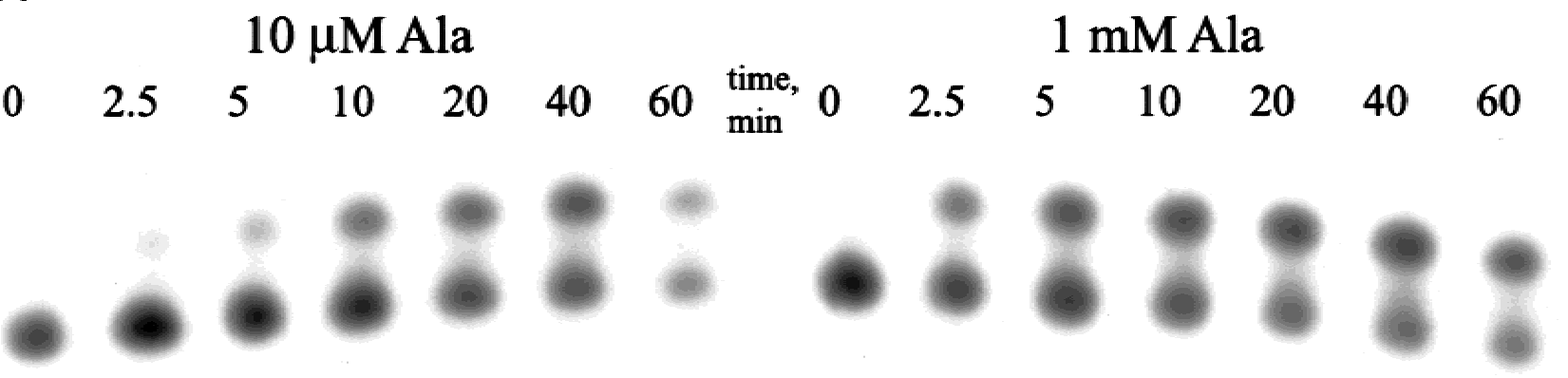

B

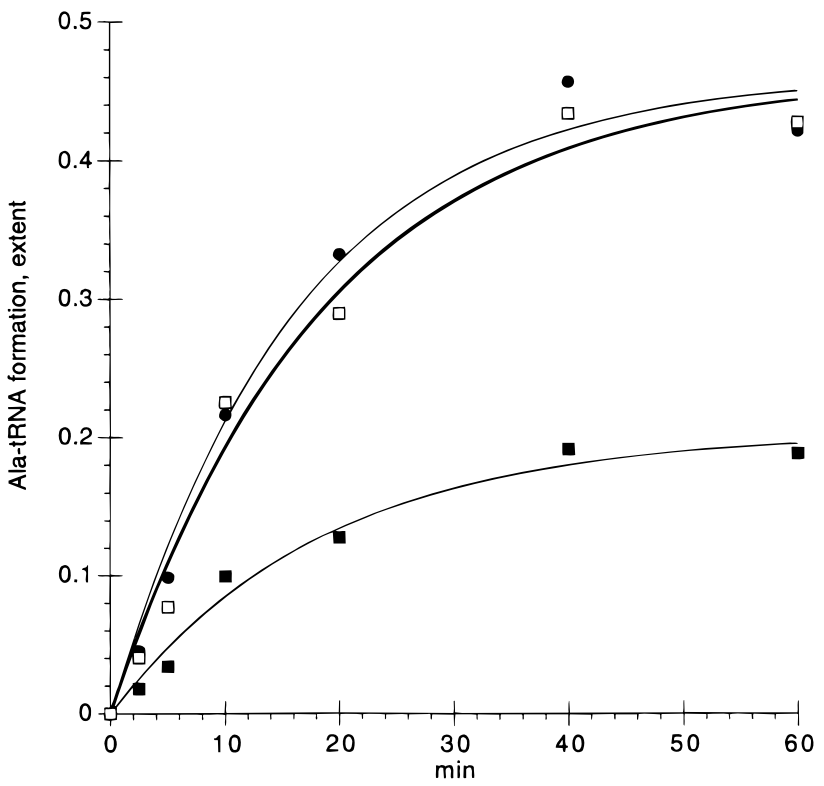

C

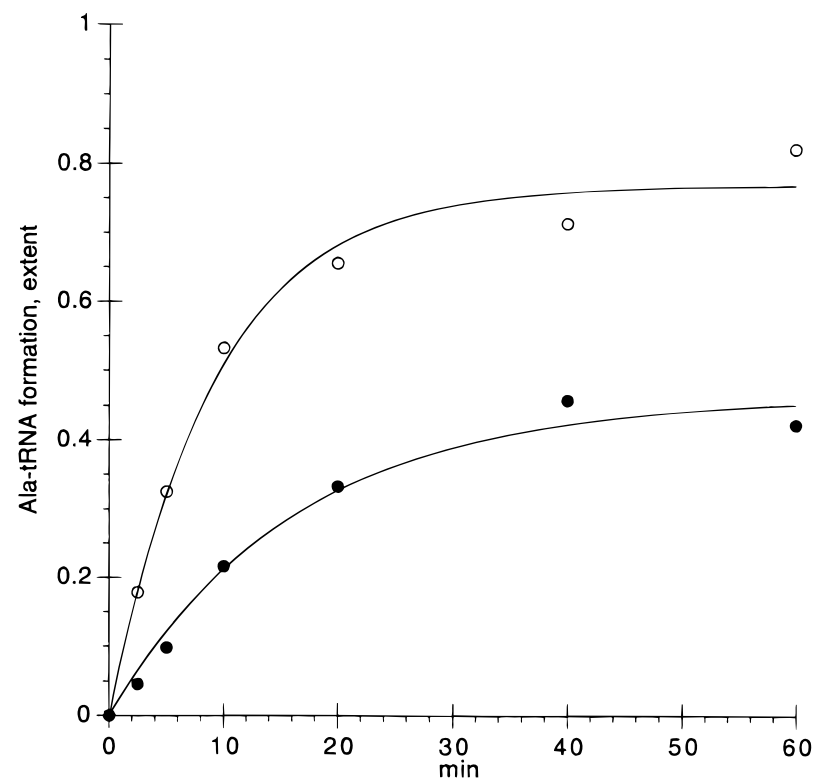

FIGURE 2. Kinetic curves of YFA2 aminoacylation measured by acid gel or by $\left[{ }^{3} \mathrm{H}\right]$-Ala incorporation. Aminoacylation was performed with $50 \mathrm{nM}$ AluRS, $1 \mu \mathrm{M}$ YFA2, and either $10 \mu \mathrm{M}$ of $\left[{ }^{3} \mathrm{H}\right]$-Ala or $1 \mathrm{mM}$ Ala. Data were fit by nonlinear least-squares regression to a standard first-order kinetic equation. A: Time course of YFA2 aminoacylation analyzed by acid gel electrophoresis. B: Aminoacylation measured in the double-label experiment either by $\left[{ }^{3} \mathrm{H}\right]$-Ala incorporation $(\boldsymbol{\square})$ or by an acid gel $(\bullet)$. Simulated curve $(\square)$ obtained by recalculating the $\left[{ }^{3} \mathrm{H}\right]$-Ala incorporation curve assuming the same extent of aminoacylation as measured by an acid gel. C: Comparison of YFA2 aminoacylation at $10 \mu \mathrm{M}(\bullet)$ and $1 \mathrm{mM}$ Ala $(\mathrm{O})$ measured by an acid gel.

absorbance in the tRNA sample, and quenching of the tritium signal during scintillation counting, potentially make this indirect assay less accurate. Indeed, when the tritium-counting efficiency is normalized such that it matches the extent of the reaction in the gel assay, the $k_{c a t}$ and $K_{M}$ (tRNA) determined by each assay are in excellent agreement (Table 1).

The incomplete aminoacylation of the nicked $\left[{ }^{32} \mathrm{P}\right]-$ labeled tRNA substrate observed in Figure 2B is either due to an inactive population of nicked tRNA molecules or to spontaneous deacylation of the aminoacyl tRNA in the reaction mixture, such that the final extent reflects a steady-state level (Bonnet \& Ebel, 1972). It appears that the latter explanation is primarily correct because, when the forward rate of aminoacylation is increased by raising the enzyme concentration from 50 $\mathrm{nM}$ to $500 \mathrm{nM}$, the extent of aminoacylation increases from $45 \%$ seen in Figure $2 \mathrm{~B}$ to $90 \%$ (data not shown). These observed extents of reaction are also consistent with the calculated rate of spontaneous deacylation of $0.1 \mathrm{~min}^{-1}$ for Ala-tRNA ${ }^{\text {Ala }}$ reported by Ott et al. (1990).

TABLE 1. Kinetic constants of YFA2 aminoacylation. ${ }^{a}$

\begin{tabular}{lcccr}
\hline & {$\left[{ }^{3} \mathrm{H}\right]$-Ala incorporation } & & \multicolumn{2}{c}{ Acid gel } \\
\cline { 2 - 2 } \cline { 5 - 5 } & $10 \mu \mathrm{M}$ Ala & & $10 \mu \mathrm{M}$ Ala & $1 \mathrm{mM} \mathrm{Ala}$ \\
\hline$K_{M}(\mu \mathrm{M})$ & $17.4 \pm 0.6$ & & $23.3 \pm 1.5$ & $15.5 \pm 3.1$ \\
$K_{\text {cat }}\left(\mathrm{min}^{-1}\right)$ & $0.73 \pm 0.18^{\mathrm{b}}$ & & $1.13 \pm 0.26$ & $3.8 \pm 0.9$
\end{tabular}

aValues for aminoacylation at $10 \mu \mathrm{M}$ Ala are obtained from the double-label experiments. Each value is an average of at least three independent experiments.

${ }^{b}$ Calculated using the extent of aminoacylation determined from the acid gel. 
If the small amount (less than $10 \%$ ) of unannealed 19 -mer is taken into account, more than $95 \%$ of the nicked tRNA substrate is active.

One of the major advantages of the gel assay is that aminoacylation kinetics can be determined under saturating concentrations of amino acid. Because the amino acid and the $3^{\prime}$ end of the tRNA occupy the same region of the synthetase, it is likely that their binding is coupled. For example, synthetases specific to Glu, Gln, and Arg do not activate the amino acid unless the tRNA is present (Arnez \& Moras, 1997; Cusack, 1997). In addition, mutations at many locations in E. coli tRNA ${ }^{\mathrm{Gln}}$ increased the $K_{M}$ for glutamine with the cognate synthetase (Ibba et al., 1996; Ibba \& Söll, 1996). By performing the gel assay at saturating and subsaturating amino acid concentrations, the importance of this coupling can be evaluated. To this end, aminoacylation kinetics were measured at $1 \mathrm{mM}$ alanine, which is well above the $K_{M}$ for Ala of $240 \mu \mathrm{M}$ determined for the first step of the reaction (Hill \& Schimmel, 1989). As shown in Figure 2C, the higher amino acid concentrations increased the initial reaction rate and, as expected, the steady-state extent of aminoacylation. The use of saturating concentrations of amino acid results in an increase in $k_{\text {cat }}$ from $1.1 \mathrm{~min}^{-1}$ to $3.8 \mathrm{~min}^{-1}$, without significant changes in $K_{M}$ (tRNA) (Table 1). More detailed kinetic analysis will be required to determine how the observed changes relate to the reaction mechanism.

The new assay requires one gel lane to separate substrate from product for each time point, which is more time consuming and somewhat more labor intensive than the traditional assay, where separation of substrate from product is done for all time points simultaneously by using a bulk washing protocol (Eigner \& Loftfield, 1974). However, the new assay uses simultaneous counting of the $\left[{ }^{32} \mathrm{P}\right]$-labeled products by a phosphorimager that is much more rapid than the sequential counting of tritium on individual filters by liquid scintillation counting. As a result, when many reaction rates must be measured, the total amount of time and effort required by the two assays is similar. In many respects, this synthetase gel assay resembles the gelbased assay for restriction enzymes and ribozymes where extensive kinetic analyses have been performed (Lesser et al., 1990; Hertel et al., 1994).

The assay presented here is more accurate than the traditional method because several assumptions and potential sources of experimental error are bypassed by directly measuring both unaminoacylated and aminoacyl tRNA. In the traditional assay, the calculation of the fraction of aminoacyl tRNA requires an accurate knowledge of the specific activity and counting efficiency of the $\left[{ }^{3} \mathrm{H}\right]$ amino acid, as well as taking reproducible aliquots of the reaction mixture. Neither of these steps enters into the determination of the fraction of aminoacyl tRNA in the gel assay, where it is simply determined as a ratio of the radioactivity in the two bands. In addition, the gel assay permits a determination of the fraction of active tRNA as detailed earlier.

The difference in gel mobility between aminoacylated and unaminoacylated tRNAs is primarily due to the extra positive charge on the amino acid, suggesting that the acid gel assay will be suitable for all tRNAsynthetase pairs. Indeed, significant gel shifts have been reported for many different intact aminoacyl-tRNAs (Varshney et al., 1991). Although we used a nicked tRNA substrate to improve resolution, it seems possible that the gel system might be improved sufficiently such that intact tRNAs can be used. Even if this is not possible, it is likely that a fully active nicked tRNA substrate can be found for any synthetase by varying the position of the nick. Many sites in yeast tRNA ${ }^{\text {Phe }}$ can tolerate a nick without altering its global folding (Pan et al., 1991), and a useful strategy has been published for finding sites in a tRNA that can be nicked without affecting aminoacylation (Aphasizhev et al., 1993). Although unmodified nicked tRNA was used in these experiments, a nick in the specific position of a modified tRNA may be prepared using deoxynucleotide-directed RNAse H cleavage (Hayase et al., 1990; Lapham et al., 1997). Additionally, whereas nonradioactive versions of all amino acids are readily available, certain radiolabeled amino acids are either unavailable or are not stable over long periods. The generality of the gel assay can therefore be considered another advantage over the traditional assay.

Finally, one of the greatest advantages of the gel assay is its much higher sensitivity. By using a [ $\left.{ }^{32} \mathrm{P}\right]$ labeled RNA substrate instead of an $\left[{ }^{3} \mathrm{H}\right]$-labeled amino acid, the gel assay can potentially detect as little as $0.01 \mathrm{fmol}$ of tRNA, making it at least 1,000 -fold more sensitive than the traditional assay. A major consequence is that this assay can be used in pre-steadystate kinetics where the enzyme is in excess of the tRNA. This permits the kinetic dissection of the tRNA binding step from the aminoacyl tRNA release step in the kinetic reaction mechanism. In preliminary experiments, we have observed aminoacylation of $40 \mathrm{fmol}$ nicked tRNA substrate by 8 pmol AlaRS, suggesting that such pre-steady-state measurements are feasible. The ability to accurately measure both the first and second steps of the aminoacylation reaction should permit the efficient determination of all the kinetic rate constants of the aminoacylation reaction by redundant steady-state and pre-steady-state methods. This information will provide a framework for the subsequent analysis of mutant proteins and tRNAs.

\section{MATERIALS AND METHODS}

Histidine-tagged E. coli AlaRS was purified from BI21-DE3 cells containing plasmid pQE-875. Cells grown to $0.6 \mathrm{OD}_{600}$ were lysed by sonication in buffer $A(50 \mathrm{mM}$ potassium phosphate, $\mathrm{pH} 7.5$, containing $50 \mathrm{mM} \mathrm{NaCl}, 10 \mathrm{mM} \beta$-mer- 
captoethanol, and $10 \%$ glycerol). Protein was purified by chromatography on a HisTrap column (Pharmacia Biotech) using the standard protocol. Fractions containing AlaRS were diluted 10-fold with buffer A and applied to a Q-Sepharose column. Protein was eluted using a linear gradient of $\mathrm{NaCl}$ in buffer A. Fractions containing AlaRS were pooled, concentrated, and stored at $-20^{\circ} \mathrm{C}$ in $50 \%$ glycerol.

The 5' fragment of YFA2 tRNA was obtained by in vitro transcription with T7 RNA polymerase (Sampson \& Uhlenbeck, 1988) from a YFA2 plasmid digested with the methylationsensitive restriction enzyme Taq $\alpha 1$ (New England Biolabs). Plasmid was obtained from the dam- E. coli strain GM-2163. The 3' fragment of YFA2 was synthesized chemically using standard procedures and phosphoramidites from Glen Research. Both fragments were purified by electrophoresis on denaturing polyacrylamide gels. For the acid gel experiments, the $3^{\prime}$ fragment was [ $\left.{ }^{32} \mathrm{P}\right]$-labeled with $\mathrm{T} 4$ polynucleotide kinase and gel-purified. The fragments were annealed by heating equimolar amounts of $5^{\prime}$ and $3^{\prime}$ fragments at $65^{\circ} \mathrm{C}$ for 2 min in $\mathrm{H}_{2} \mathrm{O}$, followed by addition of $\mathrm{MgCl}_{2}$ to a concentration of $15 \mathrm{mM}$ and subsequent slow cooling to room temperature. Stoichiometry of the annealing was verified by nondenaturing gel electrophoresis, performed at room temperature on $10 \%$ acrylamide gels containing $30 \mathrm{mM}$ Trisacetate, $\mathrm{pH} 7.5$, and $15 \mathrm{mM} \mathrm{MgCl}_{2}$.

Aminoacylation was performed in 50 mM PIPES, pH 7.0, containing $30 \mathrm{mM} \mathrm{KCl}, 10 \mathrm{mM} \mathrm{MgCl}_{2}, 1 \mathrm{mM}$ DTT, and $2 \mathrm{mM}$ ATP at $37^{\circ} \mathrm{C}$. The alanine concentration was either $10 \mu \mathrm{M}$ or $1 \mathrm{mM}$ and the AlaRS concentration was usually between 50 and $100 \mathrm{nM}$. For kinetic experiments, the tRNA concentration was varied between 0.6 and $20 \mu \mathrm{M}$. Specific activity of tRNA used in the aminoacylation experiments was in the range of $100-2,000 \mathrm{mCi} / \mathrm{mmol}$. Samples for an acid gel electrophoresis were quenched in an equal volume of freshly prepared gel loading buffer (100 mM Na-acetate, pH 5.0, 50 mM EDTA, $8 \mathrm{M}$ urea) and stored on ice.

Gel electrophoresis was performed on $10 \%$ polyacrylamide sequencing gels $(0.04 \times 40 \times 20 \mathrm{~cm})$, containing $8 \mathrm{M}$ urea and $100 \mathrm{mM}$ sodium acetate, $\mathrm{pH}$ 5.0. Gels were prepared by mixing separate stocks of $8 \mathrm{M}$ urea, $20 \%$ acrylamide in $8 \mathrm{M}$ urea, and $3 \mathrm{M} \mathrm{Na}$-acetate, $\mathrm{pH}$ 5.0. The $\mathrm{pH}$ of the mixture was always verified. Samples were applied at room temperature and gels were run for about $20 \mathrm{~min}$ at $200 \mathrm{~V}$ to allow the samples to penetrate the gel. Thereafter, gels were run at $4{ }^{\circ} \mathrm{C}$ for $12-16 \mathrm{~h}$ at $350-400 \mathrm{~V}$ until the Bromphenol Blue reached the bottom of the gel. After electrophoresis, gels were dried and quantified using a phosphorimager (Molecular Dynamics). Stability of the aminoacyl bond during electrophoresis was verified by incubation of $\left[{ }^{3} \mathrm{H}\right]$-labeled aminoacylated tRNA in the electrophoresis buffer for a period of more than $24 \mathrm{~h}$.

\section{ACKNOWLEDGMENTS}

We thank Dr. Mali Illangasekare for her helpful discussions, Dr. Andrew Feig for his careful reading of this manuscript, and Dr. Paul Schimmel for the gift of pQE-875 plasmid. This research is supported by a National Institutes of Health grant GM37552 to O.C.U.

Received May 4, 1998; returned for revision May 8, 1998; revised manuscript received May 15, 1998

\section{REFERENCES}

Aphasizhev R, Beresten S, Pugachev V, Kisselev L. 1993. Randomsplitting of tRNA transcripts as an approach for studying tRNAprotein interactions. FEBS Lett 323:175-178.

Arnez JG, Moras D. 1997. Structural and functional considerations of the aminoacylation reaction. Trends Biochem Sci 22:211-216.

Bonnet J, Ebel JP. 1972. Interpretation of incomplete reactions in tRNA aminoacylation. Aminoacylation of yeast tRNA Val II with yeast valyl-tRNA synthetase. Eur J Biochem 31:335-344.

Cusack S. 1995. Eleven down and nine to go. Nature Struct Biol 2:824-831.

Cusack S. 1997. Aminoacyl-tRNA synthetases. Curr Opin Struct Biol 7:881-889.

Eigner EA, Loftfield RB. 1974. Kinetic techniques for the investigation of amino acid: tRNA ligases (aminoacyl-tRNA synthetases, amino acid activating enzymes). Methods Enzymol 29:601-619.

Fersht AR, Mulvey RS, Koch GL. 1975. Ligand binding and enzymic catalysis coupled through subunits in tyrosyl-tRNA synthetase. Biochemistry 14:13-18.

Hayase Y, Inoue H, Ohtsuka E. 1990. Secondary structure in formylmethionine tRNA influences the site-directed cleavage of ribonuclease $\mathrm{H}$ using chimeric 2'-O-methyl oligodeoxyribonucleotides. Biochemistry 29:8793-8797.

Hertel KJ, Herschlag D, Uhlenbeck OC. 1994. A kinetic and thermodynamic framework for the hammerhead ribozyme reaction. Biochemistry 33:3374-3385.

Hill K, Schimmel P. 1989. Evidence that the $3^{\prime}$ end of a tRNA binds to a site in the adenylate synthesis domain of an aminoacyl-tRNA synthetase. Biochemistry 28:2577-2586.

Ibba M, Hong KW, Sherman JM, Sever S, Soll D. 1996. Interactions between tRNA identity nucleotides and their recognition sites in glutaminyl-tRNA synthetase determine the cognate amino acid affinity of the enzyme. Proc Natl Acad Sci USA 93:6953-6958.

Ibba M, Söll D. 1996. Protein-RNA molecular recognition [letter]. Nature 381:656.

Illangasekare M, Kovalchuke O, Yarus M. 1997. Essential structures of a self-aminoacylating RNA. J Mol Biol 274:519-529.

Lapham J, Yu YT, Shu MD, Steitz JA, Crothers DM. 1997. The position of site-directed cleavage of RNA using RNase $\mathrm{H}$ and 2'-Omethyl oligonucleotides is dependent on the enzyme source [letter; comment]. RNA 3:950-951.

Lesser DR, Kurpiewski MR, Jen-Jacobson L. 1990. The energetic basis of specificity in the Eco RI endonuclease-DNA interaction. Science 250:776-786.

Liu H, Musier-Forsyth K. 1994. Escherichia coli proline tRNA synthetase is sensitive to changes in the core region of $\mathrm{tRNA}$ (Pro). Biochemistry 33:12708-12714.

Loftfield RB. 1972. The mechanism of aminoacylation of transfer RNA. Prog Nucleic Acid Res Mol Biol 12:87-128.

Mazat JP, Merle M, Graves PV, Merault G, Gandar JC, Labouesse B. 1982. Kinetic anticooperativity in pre-steady-state formation of tryptophanyl adenylate by tryptophanyl-tRNA synthetase from beef pancreas. A consequence of the tryptophan anticooperative binding. Eur J Biochem 128:389-398.

McClain WH, Schneider J, Bhattacharya S, Gabriel K. 1998. The importance of tRNA backbone-mediated interactions with synthetase for aminoacylation. Proc Natl Acad Sci USA 95:460-465.

Ott G, Schiesswohl M, Kiesewetter S, Forster C, Arnold L, Erdmann VA, Sprinzl M. 1990. Ternary complexes of Escherichia coli aminoacyl-tRNAs with the elongation factor Tu and GTP: Thermodynamic and structural studies. Biochim Biophys Acta 1050:222225.

Pan T, Gutell RR, Uhlenbeck OC. 1991. Folding of circularly permuted transfer RNAs. Science 254:1361-1364.

Sampson JR, Uhlenbeck OC. 1988. Biochemical and physical characterization of an unmodified yeast phenylalanine transfer RNA transcribed in vitro. Proc Natl Acad Sci USA 85:10331037.

Varshney U, Lee CP, RajBhandary UL. 1991. Direct analysis of aminoacylation levels of tRNAs in vivo. Application to studying recognition of Escherichia coli initiator tRNA mutants by glutaminyltRNA synthetase. J Biol Chem 266:24712-24718.

Yarus M. 1988. tRNA identity: A hair of the dogma that bit us. Cell $55: 739-741$. 